\title{
Response to Letter to the Editor by Dr Cao regarding paper titled - "Is body-weight-supported treadmill training or robotic-assisted gait training superior to overground gait training and other forms of physiotherapy in people with spinal cord injury? A systematic review"
}

\author{
Jan Mehrholz ${ }^{1,2} \cdot$ Lisa A. Harvey $\mathbb{D}^{3} \cdot$ Simone Thomas $^{1} \cdot$ Bernhard Elsner $^{2}$ \\ Received: 28 February 2019 / Accepted: 28 February 2019 / Published online: 20 March 2019 \\ (c) International Spinal Cord Society 2019
}

We thank Dr. Cao for his interest in our paper and we are grateful for the opportunity to clarify our statistical approach to the meta-analyses in our recent systematic review [1]. Dr. Cao raises an important concern about baseline imbalance in randomised controlled trials and wonders why we did not prioritise change data over postdata in our meta-analyses [2]. The main explanation is that we worked to a protocol and in our protocol we stipulated that we would use post-data. It is not appropriate to make decisions about which data to include in meta-analyses once data extraction has commenced. This leads to bias. We opted for post-data in our protocol because these data are more commonly provided by authors. This is an important consideration particularly because it is not possible to combine post and change data in meta-analyses based on standardised mean differences [3].

It is always possible for some imbalance in some studies on some outcomes (as observed by Dr. Cao) although imbalance should only occur by chance and excessive imbalance should raise concerns about the randomisation process. We respectfully disagree with Dr. Cao's assertion that change data always provides a more robust meta-

$\triangle$ Jan Mehrholz

jan.mehrholz@klinik-bavaria.de

1 Insitute of Science, Private European Medical Academy of the Klinik Bavaria Kreischa, Kreischa, Germany

2 Department of Public Health, Dresden Medical School, Technical University Dresden, Dresden, Germany

3 John Walsh Centre for Rehabilitation Research, Kolling Institute, Sydney Medical School/Northern, University of Sydney, Sydney, Australia analysis. This is not necessarily the case and there is no consensus on this issue in the scientific literature. Cochrane Reviews typically rely on post-data [3]. Regardless, it is most unlikely that our conclusions would have changed even if we had prioritised change data in our protocol and had been able to extract change data from all included studies.

Importantly, we could have considered using change data if we could have been confident of attaining these data from the included trials. Similarly, we could have conducted more sophisticated analyses if we had access to the individual participant data of each included trial. But unfortunately, meta-analyses are limited by the data authors provide in their published papers. This limitation could have been overcome if authors provided all their individual participant data upon which their results are based in publicly available repositories or the alike (see a recent Editorial and paper in Spinal Cord encouraging all authors to archive their data for public use $[4,5])$.

Once again, we thank Dr. Cao for his thoughtful comments and for raising this important issue.

\section{Compliance with ethical standards}

Conflict of interest The authors declare that they have no conflict of interest.

Publisher's note: Springer Nature remains neutral with regard to jurisdictional claims in published maps and institutional affiliations.

\section{References}

1. Mehrholz J, Harvey LA, Thomas S, Elsner B. Is body-weightsupported treadmill training or robotic-assisted gait training superior to overground gait training and other forms of 
physiotherapy in people with spinal cord injury? A systematic review. Spinal Cord. 2017;55:722-9.

2. Cao G. Letter to the Editor: "Is body-weight-supported treadmill training or robotic-assisted gait training superior to overground gait training and other forms of physiotherapy in people with spinal cord injury? A systematic review." Spinal Cord. 2019. https://doi. org/10.1038/s41393-019-0270-8.
3. The Cochrane Collaboration. Cochrane Handbook for Systematic Reviews of Interventions Version 5. 1.0 [updated March 2011]. The Cochrane Collaboration. 2011. www.cochrane-handbook.org.

4. Dijkers MP. A beginner's guide to data stewardship and data sharing. Spinal Cord. 2019;57:169-82.

5. Dijkers M. Reduce, reuse, recycle: good stewardship of research data. Spinal Cord. 2019;57:165-6. 